\title{
ON THE SLIDING MODE CONTROL FOR PRECISION MACHINING
}

\author{
Luis Rubio $^{a^{*}}$, Asier Ibeas $^{b}$, László E. Kollár ${ }^{c}$ \\ ${ }^{a}$ ELTE, Faculty of Informatics, Savaria Institute of Technology, research fellow \\ $b$ Universitat Autonoma de Barcelona, Department of Telecommunications and Systems Engineering, associate professor \\ ${ }^{c}$ ELTE, Faculty of Informatics, Savaria Institute of Technology, full professor
}

\begin{abstract}
Precision machining uses linear motor actuators in order to deal with robustness and stability in the broad range of cutting conditions. Those systems are demanding more sophisticated control algorithms in order to fulfil each time more exigent tolerances in the tendency to miniaturization of products and to face more robust dynamics. Sliding mode control family is natural approach to cope with mass and damping variation and external disturbances, which are inherent characteristics of cutting processes. This paper compares conventional and super-twisting sliding mode controls on keeping the closed loop dynamics within requirements and reject disturbances created by the cutting forces.
\end{abstract}

Keywords: precision machining, contouring control, sliding mode control, machining dynamics

\section{Introduction}

Machining consists of the coordinated drive of multiple axis across rotational and linear movements. A central unit namely Computer Numerical Control (CNC) synchronizes the electric signals adequately to track as smooth as possible a predetermined tool path into a work-piece. Tool paths are generated in advance accordingly to the desired final product.

Commercial CNC provide feed forward and PID controllers as functionalities for end users to deal with transitory behaviour and steady state errors. Normally, these solutions provide enough accuracy, within tolerances, for the requirements of conventional processes. However, the current tendency to miniaturization results in unsatisfactory the achievements of these basic PID-based control schemes with feed forward compensation due to introduction of dynamic errors into the system.

High precision is required due to more sophisticated and precise tool paths, such as free form trajectories. Therefore, the motion of each machine axis needs to be controlled smoothly and precisely, so they will be able to follow the pre-programmed desired trajectory accurately according to the manufacturing machine task demand [1, 2].

The traditional approach to construct machine CNC has been to use rotary drive motors and ballscrews to achieve table motors. Linear motors offer several advantages over this approach, including low inertia, better performance, increased accuracy, and reduced complexity [3]. When using linear motors for machining, three variables must be considered, namely, mass, damping and disturbances acting on the system. The change of plant parameters results from the variations of mass of workpiece and damping due to use of lubricants. Furthermore, cutting forces are main source of disturbances in machining when linear motor actuators are used [4].

To overcome the influences of disturbances and model parameters uncertainties, natural control approach is adopted by sliding mode controller (SMC), which was first proposed by Utkin [5, 6]. These

(C) ELTE, Faculty of Informatics, Savaria Institute of Technology, 2020

*Corresponding author: Luis Rubio, lrr@inf.elte.hu

https://doi.org/10.37775/EIS.2020.2.4 
controllers give some desirable closed-loop properties, including invariance, dynamic order reduction, and robustness against parameters variations and disturbances. The design of SMC consists of two stages, i.e. an equivalent control and a switching control. The equivalent control is derived from the definition of sliding surface, to which the controlled system trajectories must belong. The switching control forces the system to slide along the sliding surface despite being influenced by parameter or external disturbances.

Motion control systems become vulnerable when the output tracking signals present small oscillations of finite frequency known as chattering. The chattering problem is harmful because it leads to low control accuracy, and high wear of moving mechanical parts. The chattering phenomenon can be caused by the deliberate use of classical sliding mode control technique. This control technique is characterized by a discontinuous control action with an ideal infinite frequency [7]. When fast dynamics are neglected in the mathematical model, such phenomenon can appear. Another situation responsible for chattering is due to implementation issues of the sliding mode control signal in digital devices operating with a finite sampling frequency, where the switching frequency of the control signal cannot be fully implemented [7]. A solution to this problem is the high order sliding mode technique [8]. This control technique maintains the same sliding mode properties (in this sense, first-order sliding mode) with the advantage of eliminating the chattering problem due to the continuous-time nature of the control action.

In CNC machining, SMC has been applied for controlling feed drives. A SMC applied to a CNC servomechanism was presented by Eun et al. [9]. In that work, a decoupled discrete-time SMC and disturbance compensator were proposed and applied to tune the sliding mode and disturbance estimation dynamics separately. Altintas et al. [10] proposed a Sliding Mode Controller, which compensates the cutting force and pre-compensates the frictions in drives with ball screw support bearings using a model-based approach. Altintas and Okwudire [11] developed a classical sliding mode controller with cutting force recovery to achieve high bandwidth, cutting force prediction, and active compensation of structural vibrations of the machine, but the authors concluded it was not possible of practical implementation of this approach. Although chattering will lead to deteriorate machine tool and components and limits the bandwidth and setting time of the closed-loop system it has not been taken into consideration when applying SMC in machining until the publication of $\mathrm{Xi}$ et al's work. [12, 13]. The reasons could be explained as a lack of necessity due to that the threshold of machine performance and requirements could not be achieved at that moment. Besides, these authors presented a SMC, which incorporates an integral action in order to avoid chattering of the sliding mode control. Additionally, they proposed an approach to choose a sliding surface to influence system performance and reduce chattering. However, the integral control action just mitigates the chattering problem in certain conditions and it is not a straightforward solution. Other approaches to solve the same problem for other machining processes are well documented in the literature $[14,15]$.

In this paper, the third generation of sliding mode controller (SMC), namely super-twisting sliding mode control, is proposed to tackle chattering while preserving robustness against uncertainties in the drive's parameters, compensating for external disturbances and maximizing the bandwidth within physical limitations when controlling linear motors.

Chattering is proportional to the magnitude of the discontinuous control and it will affect the activity of the control action. Super-twisting SMC algorithm inherently deals with the magnitude of the discontinuous control balancing the activity of the control action. This idea supports and enhances the use of SMC in machining to deal with model uncertainties and disturbance rejection. The comparison with conventional SMC algorithm shows the benefits of applying super-twisting SMC over conventional SMC. 
Super-twisting SMC control algorithm coping with high-precision machining processes was first proposed and addressed by the authors in a previous paper [16]. Later on, the work [17] applied successfully the algorithm to high precision machining in conventional processes in order to deal with friction of the process using rotatory DC motors. This study presents a comprehensive comparison of the conventional and super-twisting SMC approaches to high precision CNC machining in order to deal with the cutting forces of the process as disturbances in the micro-milling process using linear motors as drives.

The paper includes the following parts. Section 2 models the axis dynamics using linear motors for their application in machining and perturbations incurred by cutting forces. Section 3 presents conventional and super-twisting SMC algorithms. Section 4 summaries closed-loop composed by the system and the presented control algorithm. Section 5 presents simulation results, continuing with a discussion in section 6 and concluding in section 7 .

\section{System description}

The current section introduces the modelling of linear motors impulse by direct drives. As each motor works independently, it is intended to track-control every motor independently. The model of the linear motor direct drive feed system is established according to its work principle [11, 18]. The equation, which governs the movement of the linear motor, is a second order mass-damping equation:

$$
m \ddot{x}+b \dot{x}=F,
$$

where $x=x(t)$ denotes the position and $F$ is the external control force, $m$ is the moving mass (table and workpiece), and $b$ is the damping coefficient of the guide-ways, which is measured using traditional impact transient testing through a hammer or using other methods such as sweep frequency [19].

The block diagram is shown in Fig. 1 in which $1 /(m s+b)$ represents the Laplace transformation of Eq. 1. The movement of a single axis of the machine tool driven by linear motor is then represented by current amplifier, motor force constant, and axis dynamics including disturbance forces. In the block diagram, $u_{c}(t), i(t), F_{m}(t)$ and $F_{d}(t)$ represents the control signal, the driven current, the force given by the linear motor and the disturbance force, respectively. The index $i$ represents the integration step of the Eq. (1). Despite its simplicity, the model captures all the essence of the process and it is usually implemented in commercial CNCs.

One of the main sources of disturbance in machine tools driven by linear motors is given by the cutting forces. Previous studies have addressed the calculation of cutting forces, in which mechanistic approaches are mainly used. Different machining processes and cutting conditions lead to different

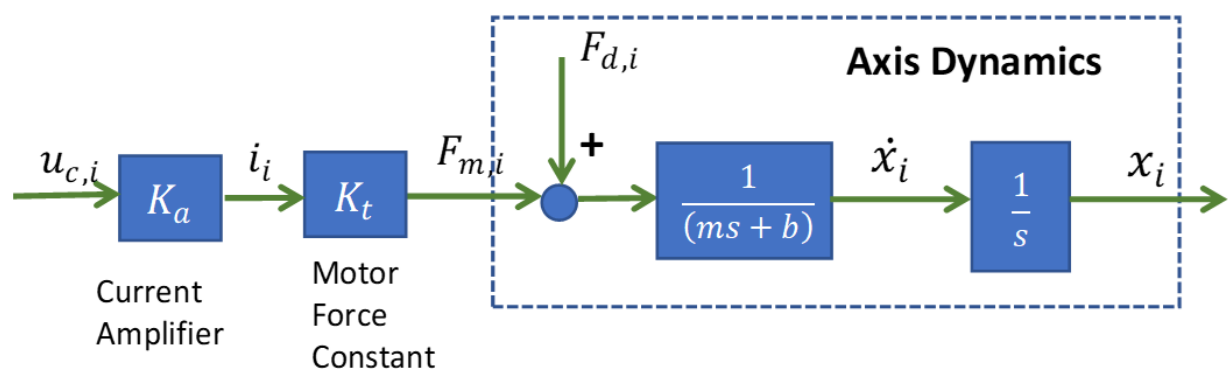

Figure 1. Block diagram of linear drive machine tool [1] 
amplitudes and frequencies of the cutting process. Furthermore, low-frequency disturbances cause dynamic stiffness between the tool and workpiece, which generates vibrations. This is still an open research topic in this field [11].

\section{Sliding mode control algorithm}

The following sections describe the design of the controller taking as initial point the system Eq. (1) for classical SMC and super-twisting SMC with PID-type sliding surface.

\subsection{Classical sliding mode control}

The control signal of the SMC is normally split out into two parts, one regarding the equivalent control, which deals with the dynamics of the system and the sliding surface, and another regarding the switching control, which is responsible for keeping the dynamics of the system onto the sliding surface of the control system. So,

$$
F=F^{e q}+F^{s w} .
$$

The equivalent control $F^{e q}$ relies on the dynamics of the system and the sliding surface, $S(t)$. The approach taken into consideration in this section is very classical and well known in the literature [7]. Consider the PID-type sliding surface:

$$
S(t)=\dot{e}(t)+\mu e(t)+\lambda \int_{0}^{t} e(\tau) d \tau .
$$

With $e(t)=x_{r e f}(t)-x(t)$, and $\mu$ and $\lambda$ are positive design parameters. Its time derivative is given by:

$$
\dot{S}(t)=\ddot{e}(t)+\mu \dot{e}(t)+\lambda e(t) .
$$

The control gains $\mu$ and $\lambda$ should be chosen so that the characteristic polynomial $s^{2}+\mu s+\lambda=0$ ( $s$ denotes a complex variable here) is strictly Hurwitz, i.e., a polynomial with roots located strictly in the open left half of the complex plane. Adequate selection of the parameters will lead to an appropriate property of the output of the system as the parameters $(\mu, \lambda)$ define the poles of the closed-loop system. The two parameters of the sliding surface are designed to trade-off the bandwidth of the system and noise rejection $\left(\lambda\left[\mathrm{rad} / \mathrm{s}^{2}\right]\right)$, and to enhance the transient response of the system $(\mu[\mathrm{rad} / \mathrm{s}])$. The control objective is imposed by $\dot{S}(t)=0, \forall t$ and $S(0)=0$, which will give the solution of the equivalent control. Thus,

$$
\dot{S}(t)=0=\ddot{x}_{r e f}(t)-\ddot{x}(t)+\mu\left[\dot{x}_{r e f}(t)-\dot{x}(t)\right]+\lambda\left[x_{r e f}(t)-x(t)\right] .
$$

Introducing Eq. (1) in Eq. (5):

$$
\ddot{x}_{r e f}(t)-\frac{1}{m}[F-b \dot{x}(t)]+\mu\left[\dot{x}_{r e f}(t)-\dot{x}(t)\right]+\lambda\left[x_{r e f}(t)-x(t)\right]=0 .
$$

While isolated, selecting $\mu=b / m$ (bandwidth of the system), the control signal can be expressed as:

$$
F=m\left[\ddot{x}_{r e f}(t)+\frac{b}{m} \dot{x}_{r e f}(t)+\lambda e(t)\right] .
$$

Since the actual parameters of the system are not known, their estimates $(\hat{m}, \hat{b})$ must be used while, in addition, a switching term is added to cope with disturbances while driving the system into the sliding surface. Therefore, the complete sliding control law reads:

$$
F=\hat{m}\left[\ddot{x}_{r e f}(t)+\frac{b}{m} \dot{x}_{r e f}(t)+\lambda e(t)\right]-\Lambda \operatorname{sign}(S), \Lambda>\delta, \delta|S|^{1 / 2}>\left|F_{d}\right| .
$$


The SMC algorithm guarantees the system reaches the sliding surface in some finite time. The disturbance is bounded by a finite positive scalar $\delta$ within the square root of the sliding surface, allowing the control law programming adequate $\Lambda$ to track the reference signal. Programming high values of $\Lambda$ introduces high frequencies to the system caused by the sign function. This element influences the control signal to be modified by chattering.

\subsection{Super-twisting sliding mode control}

In super-twisting algorithms [9], the switching control part allows reducing the chattering produced by high frequency signals while maintaining the main structure based basic features of the classical sliding mode controllers. In this case, the control law reads,

$$
F=\hat{m}\left[\ddot{x}_{r e f}(t)+\frac{b}{m} \dot{x}_{r e f}(t)+\lambda e(t)\right]-\alpha \sqrt{|S(t)|} \operatorname{sign}(S)-v(t), \alpha>0,
$$

with $\dot{v}(t)=\beta \operatorname{sign}(S), \beta>0$. The super-twisting SMC algorithm guarantees the system reaches the sliding surface in some finite time. The control gains $(\alpha, \beta)$ are selected such as the first time derivative of the corresponding disturbance, $\left(\dot{F}_{d}\right)$, is bounded by a finite positive scalar, $\gamma$. Basic requirement to tuning $(\alpha, \beta)$ is that $\beta>\gamma[7,20]$ and references therein.

This controller equation guarantees the continuity of the control law and allows avoiding/reducing the chattering compared to the classical SMC algorithm. This fact leads the system to minimize tear and wear of the actuators. Moreover, control parameters can be tuned to maximize performance indexes for production requirements, such as required tolerances and production time.

It is supposed that the reference trajectories, $x_{r e f}$, and its derivatives are known in advance since they can be obtained from the desired track and velocity requirements. With this control law, the stability of the closed loop is guaranteed in some finite time.

\section{SMC algorithms schemes}

Fig. 2 represents the integration of the whole control system consisting of the system dynamics including cutting forces as disturbances, and classical/conventional and super-twisting SMC algorithms. The simplicity of SMC methodologies does not require any further disturbance estimation and compensation but the intrinsically sign function to keep within the sliding surface under predefined disturbances bounds. References from required trajectories in position, velocity and acceleration

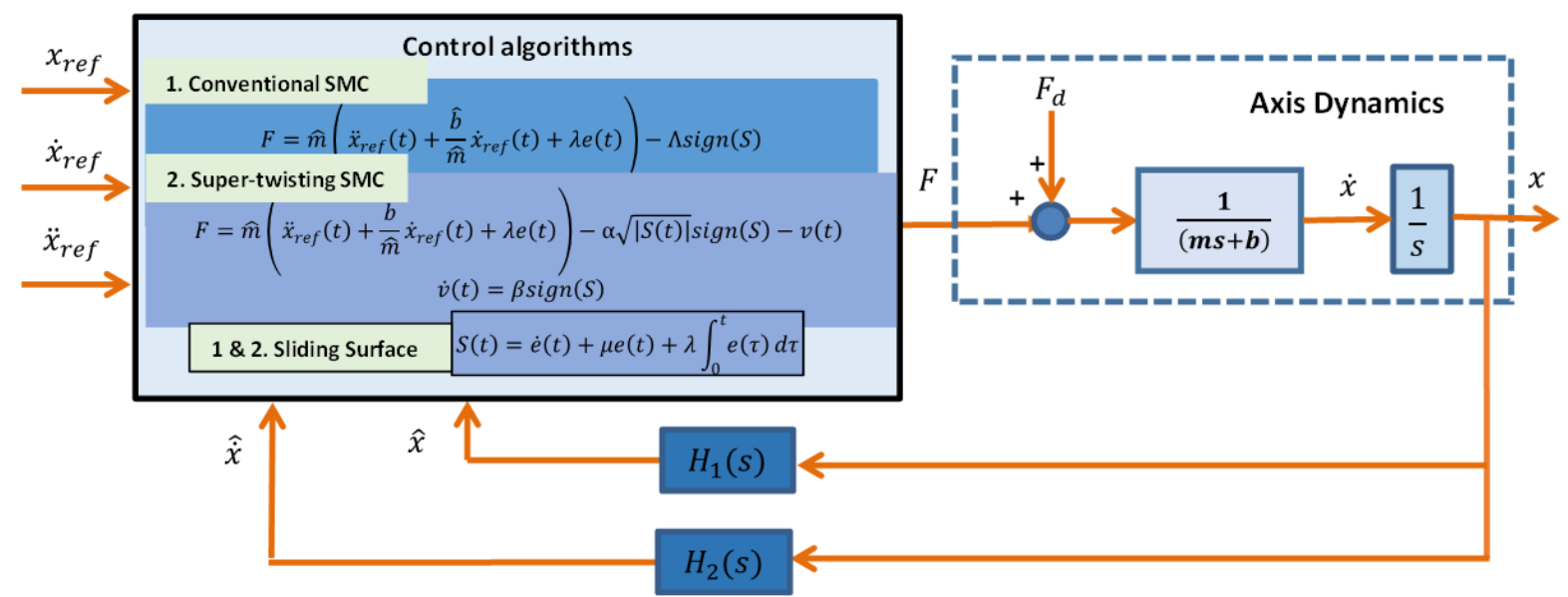

Figure 2. Closed-loop description including feed drive machine tool and SMC algorithms 
are provided from CAD/CAM systems or pre-programmed by the engineer. These trajectories may be designed in order to fulfil specifications and, to get smoothness. In order to test the proposed control algorithm sufficient excited signals in the form of sinusoidal waves are chosen as position reference signals, which are widely used in machining. Their first and second derivatives are considered as velocity and acceleration reference signals.

The control algorithm takes the reference signals with certain initial conditions for the position, velocity and acceleration and processes SMC algorithms in a certain sliding surface to generate the control signal, $F$. Before acting into the system, the control signal acquires the disturbances forces, $F_{d}$, namely cutting forces, which are inherently generated by the cutting process. The system outputs the relative position between the tool and workpiece respect to a predefined coordinate system. The position of the system is measured by an encoder, which gives an estimation of the actual value. Similarly, the velocity of the system is also estimated. Both signals are cleaned by low-pass filter in order to damp the influence of high frequencies. In the following loop, the sliding surface and the control algorithms are processed again, keeping the output within the sliding surface while respecting the predefined tolerance despite the disturbances acting on the system. The loop is repeated at every control time interval.

\section{Results}

The simulations were implemented in Matlab/Simulink environment using the integrated numerical method for solving the Eq. (1)-(9) in continuous time. Continuous time domain simulations are typical approaches in control systems theory in order to analyse the behaviour of the system.

The simulations consist of reference inputs in form of sinusoidal waves, which are movements along individual axes, sinusoidal disturbance due to cutting parameters according to micro-milling processes, and variation of mass of the workpiece due to a material removal process and damping in the process due to the use of lubricants when machining.

Extensive simulations have been carried out in order to evaluate the proposed control algorithm. In the simulations, a sinusoidal reference input signal of amplitude one millimetre (or $2 \mathrm{~mm}$ peak to peak) and $3 \mathrm{rad} /$ second of frequency is used to test the control. Disturbances correspond to cutting forces in 2 teeth and $0.9 \mathrm{~mm}$ diameter micro-milling tool at $11520 \mathrm{rpm}, 0.9 \mathrm{~mm}$ axial depth of cut and $1.08 \mathrm{~mm} / \mathrm{min}$ feed rate, which are typical in micro milling processes [21].

The following parameters and assumptions have been taken into consideration in the simulation, actual mass and damping $m=10 \mathrm{~kg}, b=250 \mathrm{~kg} / \mathrm{s}$, and amplifier and motor force constant $K_{t}=2 \mathrm{~A} / \mathrm{V}, K_{a}=50 \mathrm{~N} / \mathrm{A}$. The parameters of the plant change slightly with time due to variations in the mass during the cutting process, variations in the damping due to lubricant and due to uncertainties in their values. In the simulations the estimated mass is considered as $\hat{m}=7 \mathrm{~kg}$ and the estimated damping $\hat{b}=200 \mathrm{~kg} / \mathrm{s}$.

Individually, the control performance hinges on the selection of parameters of the sliding surface, $(\mu, \lambda)$, how well the parameters of the transfer function have been estimated, namely mass and damping and, the selection of the constant of sign function on the control $\Lambda$, for the SMC and the pairs $(\alpha, \beta)$ for the super-twisting SMC. In this section, just the variation of the sign function parameter $\Lambda$, for the SMC and the pairs $(\alpha, \beta)$ for the super-twisting SMC, are considered to compare both controllers, the other parameters were adjusted as mentioned before and sliding surface parameters are selected as $(\mu, \lambda)=(2,1)$. For each simulated scenario, four integrated figures are plotted. The upper left figure plots the reference position in dashed red and the actual position in blue, the second one represents the position error, and the third one outputs velocity error and the fourth one is the control signal. 

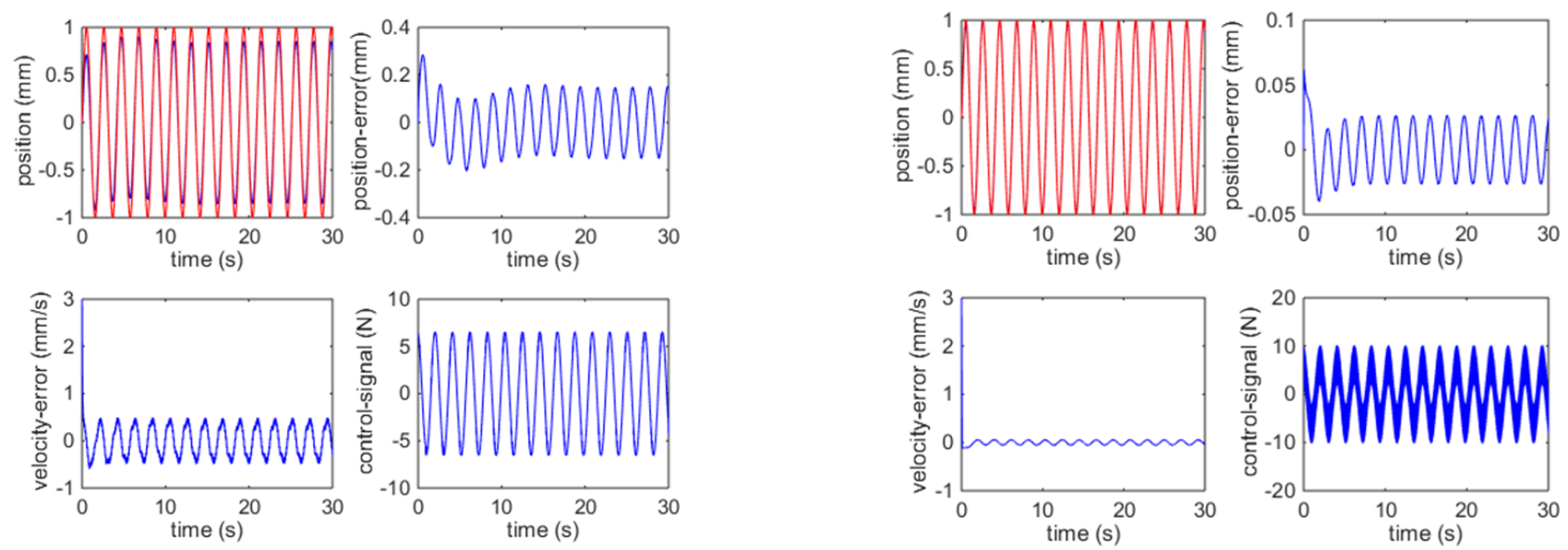

(a)

(b)

Figure 3. a) Outputs SMC with $\Lambda=40, b)$ Outputs SMC with $\Lambda=400$

Fig. 3(a) represents the SMC with $\Lambda=40$ under the influence of the cutting force acting as external disturbance. It can be shown that the control signal is smooth but the tracking error is significant, leading to the system not to follow pre-established tool path references.

In order to deal with error the parameter $\Lambda$ is increased to 400, as it can be seen in Fig. 3(b). In this case, adequate tracking performance is obtained, however chattering due to high frequencies introduced by the sign function in the control signal appears, as it can be shown in the control signal plot of the Fig. 3(b). The trade-off between precision in tracking the reference signal and chattering limits the control algorithm. To deal with chattering problem, super-twisting SMC has been applied. Fig. 4(a) shows that better performance in tracking the reference signal can be achieved without introducing chattering in the control signal. Balance between tuning control parameters to reach adequate tracking and chattering is required to achieve satisfactory performance indexes. High values of control parameters makes the algorithms more demanding in terms of control power, i.e. amplifiers, and more sensitive to introduce noise to the system.
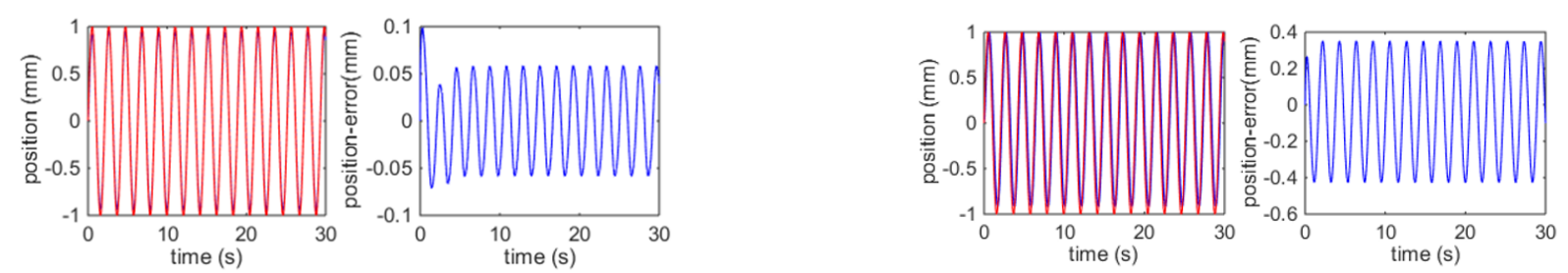
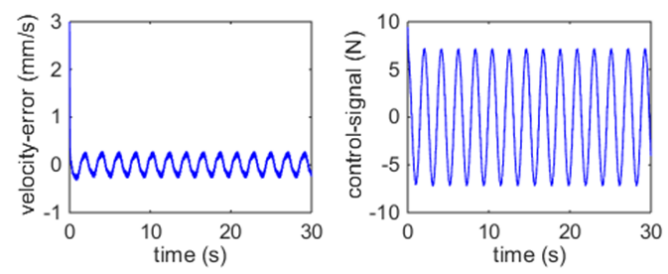

(a)

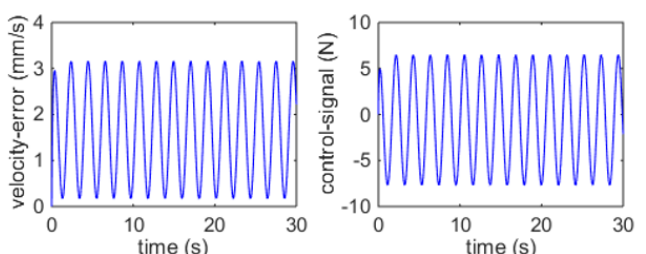

(b)

Figure 4. a) Outputs Super-twisting $\operatorname{SMC}$ with $(\alpha, \beta)=(200,200)$, b) Outputs commercial CNC 

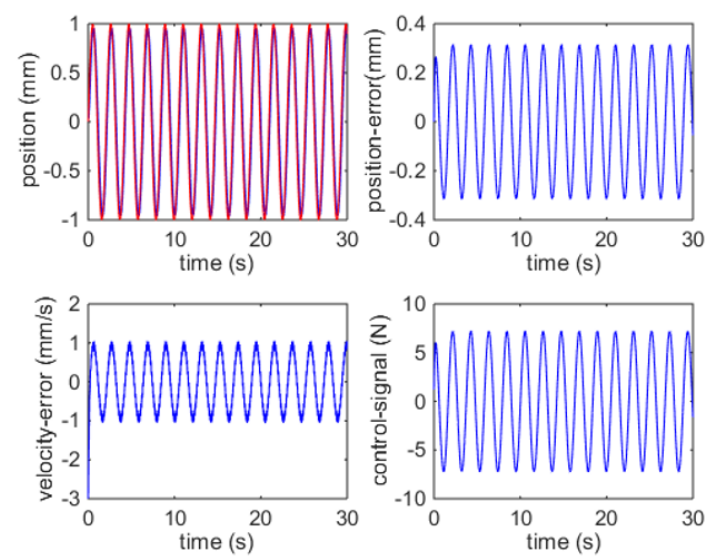

Figure 5. Outputs P-PD cascaded control system

In order to compare the proposed algorithmic methodologies with conventional PID controllers, other two controllers are exposed in this section. The first one is a typical control loop implemented in a state of the art of commercial CNC (Fig. 4(b)), and the second one is the P-PD cascaded control loop [16] (Fig. 5). As figures show, the position or tracking error is considerably appreciated in PID based controllers. In shop floors, these errors are compensated before machining.

\section{Discussion and future research paths}

Currently, CNCs host PID algorithms to face the tracking control of precision machining. The tendency of precision machining is to achieve each time more accuracy and better tracking performance in machining. The performance of motion of PID control algorithms may be significantly deteriorated by the nonlinear plant uncertainties and unknown external disturbances, compensation of dynamic errors is needed to achieve certain degree of quality [22], so adequate PID-based controllers only achieve limited performance when applied to precision machining. Furthermore, free form trajectories, which introduce high frequency inputs, are required in these processes. The realization of free form trajectories in machining leads to reduced product errors, improving accuracy, and minimize time and costs of the whole process. The programming of these trajectories makes the PID controllers inappropriate for the next generation of machining processes. The SMC algorithms have been exhibited promising performance to deal with these systems and they enhanced previous results.

The PID-type sliding surface controls the bandwidth and setting time of the system, however, increasing the bandwidth of the system will lead to the system to be exposed to noise and acquire vibration frequencies due to external perturbations. A trade-off between bandwidth and rejection of noise and filter frequencies which excites machine tool structure are required. The super-twisting algorithm allows keeping the bandwidth of the system to a maximum value while rejecting noise and disturbance frequencies, which excites the machine tool structure, but it is not influenced by chattering in the control signal compared to classical SMC.

As a result, the following future research paths are considered:

1. Discretise the algorithm to be implemented, testing the feasibility of the provided solution in a test based environment.

2. Propose alternative switching control algorithms, which suits with the problem of machining in order to reject disturbances and follow inputs with high frequency sinusoidal components.

3. Provide intelligent skills to accommodate requirements of the process. 


\section{Conclusions}

In this paper, the super-twisting sliding mode control algorithm with PID-type sliding surface is proposed to deal with the motion tracking of linear motors actuated in micro-milling processes. The micro-milling cutting acts as disturbances into the linear motor, which is composed by mass and damper against inertia of the system. The following key findings can be pointed out:

- Sinusoidal motion tracking simulations demonstrate that the proposed super-twisting SMC controller may improve the performance tracking compared with SMC.

- The PID-type sliding surface balances the bandwidth of the system and settling time response while rejects noise and vibratory frequencies into the system.

- The proposed controller is able to achieve sub-micron accuracy single axis motion tracking depending upon the resolution positioning sensor. The higher resolution the sensor has, the better positioning may be achieved.

\section{Acknowledgement}

The research was carried out in the frame of the project "EFOP-3.6.1-16-2016-00018 - Improving the role of research + development + innovation in the higher education through institutional developments assisting intelligent specialization in Sopron and Szombathely". A. Ibeas is grateful to the Basque Government for its support through grant IT1207-19.

\section{References}

[1] Y. Altintas, Manufacturing Automation: Metal Cutting Mechanics, Machine Tool Vibrations, and CNC design 2nd Ed., Cambridge University Press, 2012, pp. 250-312. CrossRef

[2] C-H. Yeung, Y. Altintas, K. Erkorkmaz, Virtual CNC system. Part I: System Architecture, International Journal of Machine Tools and Manufacture 46(10), 2006, pp. 1107-1123. CrossRef

[3] S. Gordon, M.T. Hillery, Development of a high-speed CNC cutting machine using linear motors, Journal of Materials Processing Technology 166(3), 2005, pp. 321-329. CrossRef

[4] M.S. Heydarzadeh, S.M. Rezaei, N.A. Mardi, A. Kamali E, Motion control of a two-axis linear motor-driven stage in the micro-milling process, Proc. Inst. Mech. Eng. Part E 232(1), 2016. pp. 65-76. CrossRef

[5] V.I. Utkin, Sliding modes in control and optimization, Springer-Verlag, Berlin, 1992, CrossRef

[6] V.I. Utkin, Sliding mode control design principles and application to electric devices, IEEE Transactions Industrial Electronics 40(1), 1993, pp. 23-36. CrossRef

[7] J. Rivera, L. Garcia, C. Mora, J.J. Raygoza, S. Ortega, Super-Twisting Sliding Mode in Motion Control Systems, In: Andrzej Bartoszewicz, Sliding Mode Control, IntechOpen, 2011, Chapter 13, pp. 237-254. CrossRef

[8] A. Levant, Quasi-continuous high-order sliding-mode controllers, IEEE Transactions on Automatic Control 50(11), 2005, pp. 1812-1816. CrossRef

[9] Y. Eun, J. Kim, K. Kim, D. Cho, Discrete-time variable structure controller with a decoupled disturbance and its application to a CNC servomechanism, IEEE Transactions on Control Systems Technology 7(4), 1999, pp. 414-423. CrossRef 
[10] Y. Altintas, K. Erkorkmaz, W-H. Zhu, Sliding mode controller for high-speed feed drives, Annals of the CIRP 49(1), 2000, pp. 265-270. CrossRef

[11] Y. Altintas, C.E. Okwudire, Dynamic stiffness enhancement of direct-driven machine tools using sliding mode with disturbance recovery, Annals of the CIRP 58(1), 2009, pp. 335-338. CrossRef

[12] X-C. Xi, W-S. Zhao, A-N. Poo, Improving CNC countering accuracy by robust digital integral sliding mode control, International Journal of Machine Tools and Manufacture 88, 2015, pp. 51-61. CrossRef

[13] X-C. Xi, G-S. Hong, A-N. Poo, Improving CNC contouring accuracy by integral sliding mode control, Mechatronics 20(4), 2010, pp. 442-452. CrossRef

[14] C. Choi, T-C. Tsao, Control of linear motors for machine tool feed drives: experimental investigation of optimal feed-forward tracking control, Journal of dynamic systems, measurement and control 120(1), 1998, pp. 137-142. CrossRef

[15] C. Choi, T-C. Tsao, Control of linear motor machine tool feed drives for end-milling: Robust MIMO approach, Mechatronics 15(10), 2005, pp. 1207-1224. CrossRef

[16] L. Rubio, A. Ibeas, X. Luo, P-PI and super twisting sliding mode control schemes comparison for high-precision CNC machining, 24th Iranian Conference on Electrical Engineering (ICEE), Shiraz, 2016, pp. 1825-1830. CrossRef

[17] D. Papageorgiou, M. Blanke, H.H. Niemann, J.H. Richter, Adaptive and sliding mode friction resilent machine tool positioning - Cascaded control revisited, Mechanical Systems and Signal Processing 132(1), 2019, pp. 35-54. CrossRef

[18] F.J. Villegas, R.L. Hecker, M.E. Pena, D.A. Vicente, G.M. Flores, Modelling of a linear motor feed drive including pre-rolling friction and aperiodic cogging and ripple, The International Journal of Advanced Manufacturing Technology 73, 2014, pp. 267-277. CrossRef

[19] P.J. Torvik, On estimating system damping from frequency response bandwidths, Journal of Sound and Vibration 330(25), 2011, pp. 6088-6097. CrossRef

[20] X. Yu, M.Önder, Recent advances in sliding modes: from control to intelligent mechatronics, Studies in systems, decision and control, Springer, 2015.

[21] B. Wu, Analysis and simulation of micro-milling processes, Master Engineering Thesis, University of South Wales, Faculty of Engineering, 2013.

[22] C. Liu, S. Xiang, C. Lu, C. Wu, Z. Du, J. Yang, Dynamic and static error identification and separation method for three-axis CNC machine tools based on feature workpiece cutting, The International Journal of Advanced Manufacturing Technology 107, 2020, pp. 2227-2238. CrossRef 